\title{
Distribución geográfica de los sismos contenidos en el catálogo de la Red Sismológica Nacional de Costa Rica
}

\section{Geographic distribution of the earthquakes included in the catalog of National Seismological Network of Costa Rica}

\author{
Kevin Godinez-Rodríguez ${ }^{1}$ \\ Mario Arroyo-Solórzano ${ }^{2}$ \\ Lepolt Linkimer-Abarca ${ }^{3}$ \\ Universidad de Costa Rica, Costa Rica
}

http://dx.doi.org/10.15359/rgac.60-1.6

\section{RESUMEN}

En este artículo se analiza la distribución espacio-temporal de la sismicidad en Costa Rica, contenida en el catálogo sísmico de la Red Sismológica Nacional para el periodo 1975-2014. La distribución geográfica de la sismicidad resalta las principales estructuras tectónicas activas del país. En particular, cúmulos de sismicidad revelan zonas de alta deformación cortical ubicadas sobre la proyección hacia el continente de montes submarinos y de la Zona de Fractura de Panamá. Estos cúmulos no concuerdan exactamente con las zonas de mayor liberación de energía sísmica. Se detecta, además, un "vacío de sismos" que corresponde con parte de la Cordillera de Talamanca. La inspección de la sismicidad con base en la división político administrativa muestra que el cantón con mayor cantidad de sismos es Pérez Zeledón, mientras que los cantones con la densidad más alta de sismos son: Parrita, El Guarco, Tarrazú, León Cortés y Desamparados.

1 Estudiante de Geografia, Universidad Nacional y Asistente, Escuela Centroamericana de Geología, Universidad de Costa Rica. Apdo. 214-2060, San Pedro, Costa Rica. Correo: kevin.godinezrodriguez@ucr.ac.cr

2 Bachiller en Geografía y Geología. Asistente en Escuela Centroamericana de Geología, Universidad de Costa Rica. Apdo. 214-2060, San Pedro, Costa Rica. Correo: mario.arroyosolorzano@ucr.ac.cr

3 Profesor catedrático, Escuela Centroamericana de Geología y Red Sismológica Nacional, Universidad de Costa Rica. Apdo. 214-2060, San Pedro, Costa Rica. Correo: lepolt.linkimer@ucr.ac.cr 
Kevin Godinez-Rodríguez, Mario Arroyo-Solórzano, Lepolt Linkimer-Abarca. Geographic distribution of the earthquakes included in the catalog of National Seismological Network of Costa Rica

Palabras clave: Sismicidad en Costa Rica, catálogo sísmico, densidad de sismos.

\begin{abstract}
This article analyzes the time-geographic distribution of earthquakes in Costa Rica for the seismicity included in the catalog of the Red Sismológica Nacional (National Seismological Network) during 1975-2014. The geographic distribution of seismicity highlights the main active tectonic structures. In particular, clusters of seismicity reveal highly deformed crustal areas, which are located along the inland projected path of seamount chains and the Panama Fracture Zone. These clusters do not correlate exactly with the largest energy released zones. A seismic gap is also detected in the Talamanca mountain range. The inspection of seismicity based on the political division of the country shows that the county with the largest amount of recorded earthquakes is Pérez Zeledón, and the counties with the highest earthquake density are: Parrita, El Guarco, Tarrazú, León Cortés, and Desamparados.
\end{abstract}

Keywords: Seismicity of Costa Rica, seismic catalog, density of earthquakes.

\title{
Introducción
}

La Red Sismológica Nacional (RSN: UCR-ICE) es uno de los entes dedicados a la vigilancia sísmica de Costa Rica. Fue creada mediante un convenio de cooperación técnica y científica entre la Sección de Sismología, Vulcanología y Exploración Geofísica (SSVEG) de la Escuela Centroamericana de Geología de la Universidad de Costa Rica (UCR) y el Área de Amenazas y Auscultación Sísmica y Volcánica (AAASV) del Instituto Costarricense de Electricidad (ICE). La SSVEG y el AAASV funcionan desde 1973 y 1974, respectivamente, y el centro de registro conjunto de la RSN, con sede en la UCR, opera desde 1982.

El catálogo sísmico de la RSN analizado en este estudio está compuesto por 111.850 sismos que ocurrieron entre el 4 de noviembre de 1974 y el 31 de diciembre del 2014. Este catálogo incluye las características básicas de los sismos localizados, es decir: la fecha, hora, latitud y longitud del epicentro, profundidad del hipocentro y en la mayoría de los casos su magnitud. La cantidad y distribución de las estaciones con las que se han localizado los sismos de este catálogo han variado significativamente a lo largo de la historia de la RSN. En 1976, la red de estaciones contaba con únicamente cinco instrumentos en la parte central de Costa Rica (Morales, 1986), número que creció paulatinamente hasta alrededor de 60 estaciones, ubicadas en todo el país para el 2014.

En este artículo se analiza la distribución geográfica de la sismicidad contenida en el catálogo sísmico de la RSN en Costa Rica para el periodo de tiempo 1975-2014. El objetivo del estudio es brindar una idea 
de la completitud del catálogo actual y de las zonas que reflejan la mayor sismicidad y la mayor liberación de energía sísmica. Asimismo, se hace una inspección por cantones para determinar cuáles son los que tienen la mayor o menor cantidad de sismos. La identificación de estas zonas con mayor sismicidad y liberación de energía sísmica en las diferentes regiones del país es importante como insumo para estudios de amenaza sísmica de una región particular y, para caracterizar la sismicidad registrada en Costa Rica.

\section{Área de estudio}

El área de estudio se enfoca en Costa Rica, para lo cual, se toma una zona de influencia de sectores de países aledaños (sur de Nicaragua y norte de Panamá), y el Océano Pacífico y Mar Caribe. Dado que el catálogo de la RSN contiene sismos regionales (a nivel Centroamericano) y telesismos (terremotos a nivel global), se restringió el área de estudio para enfocar el análisis de la sismicidad dentro del territorio de Costa Rica. De esta forma, se analizaron, únicamente, los sismos ubicados entre las coordenadas de latitud que van desde $7^{\circ} \mathrm{N}$ a $11,5^{\circ} \mathrm{N}$ y longitud desde $82^{\circ} \mathrm{W}$ a $86,5^{\circ} \mathrm{W}$. Esta porción del catálogo está compuesta por 68.022 sismos.

Costa Rica se caracteriza por una alta sismicidad, debido a su localización en una zona de subducción, en donde interactúan las placas del Coco, Caribe y Nazca y la microplaca de Panamá (Mapa 1). La sismicidad es especialmente abundante a lo largo de la Fosa Mesoamericana y en la zona sismogénica interplacas, donde se subduce la placa del Coco debajo de la placa Caribe y la microplaca de Panamá (Montero, 2001). Además, la sismicidad es alta a lo largo de la Zona de Fractura de Panamá (ZFP), la cual, sirve de límite entre las placas del Coco y Nazca y a lo largo del Cinturón Deformado del Norte de Panamá (CDNP) y el Cinturón Deformado del Centro de Costa Rica (CDCCR), los cuales son anchas zonas de deformación cortical con una alta densidad de fallas activas (e.g., Fan y otros., 1993; Goes y otros., 1993, Marshall y otros., y 2000, Montero, 2001), que han sido postulados como límites entre la microplaca de Panamá y la placa Caribe (Mapa 1). 
Kevin Godinez-Rodriguez, Mario Arroyo-Solórzano, Lepolt Linkimer-Abarca. Geographic distribution of the earthquakes included in the catalog of National Seismological Network of Costa Rica

Mapa 1: Mapa tectónico simplificado de Costa Rica y ubicación de las estaciones de la RSN durante el año 2014

Costa Rica, Mapa Tectónico Simplificado

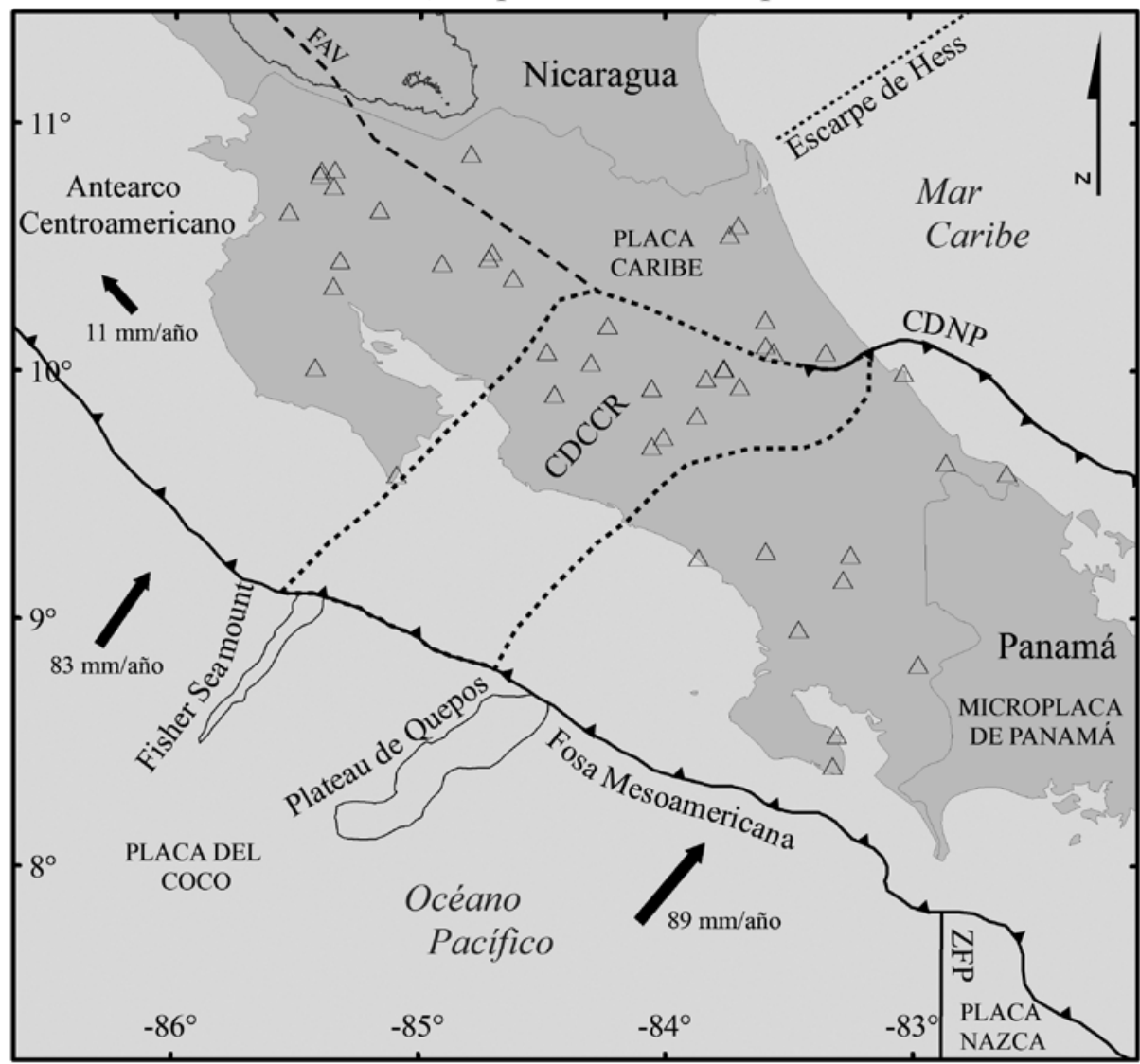

\section{Simbología}

$\triangle$ Estaciones RSN

....... Escarpe de Hess

—_ Fosa Mesoamericana

- - - Fallas del arco volcánico (FAV)

— Zona de fractura de Panamá (ZFP)

$\checkmark$ Cinturón deformado del norte de Panamá (CDNP)

.... Cinturón deformado del centro de Costa Rica (CDCCR)

\section{Fuentes:}

RSN (UCR-ICE).

Atlas 2014 del ITCR.

Montero \& Rojas (2014)
Datum y Esferoide: WGS84. Proyección: CRTM05.
Diagrama de Ubicación

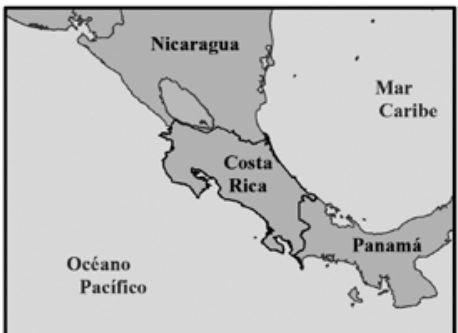

Elaboró: Mario Arroyo,

Kevin Godinez \& Lepolt Linkimer Febrero, 2016 


\section{Marco teórico-conceptual}

En esta sección se comentan brevemente dos conceptos relevantes para el desarrollo de este estudio: la completitud de un catálogo sísmico y la energía sísmica. El catálogo sísmico es uno de los productos más importantes que una red sismológica puede aportar a la comunidad científica y a la sociedad. La magnitud de completitud (MC) de un catálogo sísmico se define como la magnitud más baja en la cual el 100\% de los sismos son detectados exitosamente dentro de una región y periodo de tiempo (e.g., Woessner y Wiemer, 2005). MC depende de muchos factores que afectan la capacidad de detección de una red sismológica, como son: la densidad y distribución de estaciones sísmicas, el tipo de instrumentación usada, la eficiencia del envío de datos desde las estaciones al centro de procesamiento, las prácticas y procedimientos de detección de sismos, entre otros (e.g., Chouliaras, 2009; Mignan y Woessner, 2012).

Una manera para determinar la MC es a través de la metodología de Stepp (1972). Este método evalúa la estabilidad de la tasa media de la ocurrencia de sismos en un rango de magnitud y en una serie de periodos de tiempo predefinidos. Este método supone que la tasa de ocurrencia de los sismos en una región es uniforme o se mantiene constante a través del tiempo. Además, infiere que la ocurrencia de terremotos sigue una distribución de Poisson, es decir, que hace referencia a variables sometidas a influencias, impactos o fenómenos aleatorios que evolucionan en función de un índice, que puede ser el tiempo o el espacio (e.g., Cárdenas, Garzón, Santa y Castillo, 2010).

La energía sísmica, por otro lado, es un concepto que se relaciona con la posibilidad de daños a las estructuras hechas por el hombre. Teóricamente, su cálculo requiere la suma del flujo de energía en un amplio conjunto de frecuencias generadas por un terremoto durante la ruptura. $\mathrm{La}$ mayoría de las estimaciones de la energía se han basado históricamente en la relación empírica desarrollada por Gutenberg y Richter (1956): $\log 10 \mathrm{E}$ $=11,8+1,5$ Ms. En esta relación, la energía, E, se expresa en ergios, y Ms es la magnitud de las ondas superficiales (Spence, Sipkins y Choy., 1989).

Cuando se produce un evento sísmico, una fracción de la energía de deformación acumulada en la roca es irradiada en forma de ondas sísmicas. Convencionalmente, se emplea la escala de magnitud como método comparativo para demostrar la cantidad de energía liberada por los 
Kevin Godinez-Rodríguez, Mario Arroyo-Solórzano, Lepolt Linkimer-Abarca. Geographic distribution of the earthquakes included in the catalog of National Seismological Network of Costa Rica

terremotos grandes y pequeños. Por cada aumento en magnitud en una unidad, la energía sísmica asociada aumenta cerca de 32 veces (Spence et al., 1989). A partir de mediciones realizadas de la energía de las ondas sísmicas producidas durante los terremotos, se estima que la energía liberada por año en todo el mundo oscila entre 1025 y 1026 ergios (Bolt, 1993).

\section{Estado de la cuestión}

Para Costa Rica, existen algunos estudios que se adentran en la inspección geográfica de un catálogo sísmico. Por ejemplo, están los realizados por el Observatorio Vulcanológico y Sísmico de Costa Rica de la Universidad Nacional (OVSICORI-UNA) en los que se detallan los sismos más significativos que han ocurrido durante la existencia de ese centro (e.g., Segura, Quintero, Burgoa y Jiménez, 2014). En el caso de la RSN, existen algunos estudios, como el de Rojas, Cowan, Lindholm, Dahle y Bungum (1993), en el cual se determinaron ecuaciones para la conversión de un tipo de magnitud a otro y los parámetros de la Ley de Gutenberg-Richter para Costa Rica.

Un estudio más reciente que presenta estrecha relación con esta investigación es el de Linkimer y Alvarado (2014), el cual muestra algunos datos estadísticos de los sismos sentidos contenidos en el catálogo bajo estudio. Dentro de los resultados que presentan, sobresale que la cantidad anual de sismos sentidos reportados por la RSN ha variado entre 15 y 353 y que la mayor cantidad de sismos sentidos reportados por esa red ocurrió en los años 1991, 2012 y 2013, lo cual estuvo relacionado con la ocurrencia de terremotos importantes, sus réplicas y sismos inducidos durante esos años.

De manera regional, Benito (2008) trabaja los catálogos disponibles para Centro América y determina la relación Gutenberg-Richter y la MC. También para Latinoamérica, existen otros estudios similares en Venezuela (Valladares, 2013), Colombia (Caneva Salcedo, van Heissenoven y Alfaro, 2003) y México (Benito y Jiménez, 1999). En Estados Unidos y Europa algunas referencias importantes en el tema son: Woessner y Wiemer (2005); Ranjit, Wason y Sharma (2012); Mignan y Woessner (2012) y Chouliaras (2009). 


\section{Marco metodológico}

\section{El catálogo de sismos de la RSN}

Con el fin de descartar los sismos con localizaciones de baja calidad contenidos en el catálogo, se tomaron en cuenta algunos criterios. Por ejemplo, se obviaron del análisis los eventos localizados con menos de cuatro estaciones y también, los caracterizados por un valor de la raíz cuadrática media (RMS) mayor a 1,0. Tampoco fueron tomados en cuenta aquellos que no contaban con una estimación de magnitud. Adicionalmente, se realizó una inspección de la profundidad, y fueron descartados aquellos sismos con profundidades atípicas, considerando el espesor de la corteza y profundidad de la losa de la placa del Coco que se subduce bajo Costa Rica (Lücke, 2012). El catálogo filtrado de acuerdo con las restricciones y los criterios mencionados, está compuesto por 62.514 sismos (Cuadro 1).

Cuadro 1: Cantidad de sismos en rangos de cinco años del catálogo de la RSN

\begin{tabular}{|c|c|}
\hline Intervalo de tiempo & $\begin{array}{c}\text { Cantidad total de sismos } \\
\text { registrados }\end{array}$ \\
\hline $2010-2014$ & 9.689 \\
\hline $2005-2009$ & 13.664 \\
\hline $2000-2004$ & 16.705 \\
\hline $1995-1999$ & 10.709 \\
\hline $1990-1994$ & 8.389 \\
\hline $1985-1989$ & 2.465 \\
\hline $1980-1984$ & 733 \\
\hline $1975-1979 \quad$ Total & 160 \\
\hline
\end{tabular}

Fuente: Elaboración propia con base en el catálogo de sismos de la RSN.

Otro aspecto tratado en la preparación del catálogo fue la uniformización de la magnitud, ya que la determinación de la misma ha variado a lo largo de la historia de la RSN. Antes del 2012, se determinaba principalmente la magnitud coda (Md) o la magnitud local (ML). Sin embargo, desde el 2012, se estima únicamente la magnitud momento (Mw). Con el fin de realizar un análisis homogenizado de la magnitud del catálogo, se 
Kevin Godinez-Rodríguez, Mario Arroyo-Solórzano, Lepolt Linkimer-Abarca. Geographic distribution of the earthquakes included in the catalog of National Seismological Network of Costa Rica

procedió a convertir las magnitudes ML y Md a Mw. Para la conversión de las magnitudes, se usaron las relaciones determinadas por Rojas (1993) y Rojas et al. (1993).

El catálogo de la RSN se analizó para años completos, es decir, comenzando el 1 de enero de 1975 y hasta el 31 de diciembre del 2014. Se realizó una inspección de la sismicidad para intervalos de tiempo de cinco años desde 1975 al 2014, es decir, se consideraron los siguientes intervalos de tiempo: 1975-1979, 1980-1984, 1985-1989, 1990-1994, 1995-1999, 2000-2004, 2005-2009 y 2010-2014 (Figuras 1 y 2). Además, se trabajó con 12 rangos de magnitud, que incluyen un rango de $\mathrm{Mw} \leq 1,9$, diez rangos desde 2,0 a 6,9 subdivididos en intervalos de 0,5 (i.e., 2-2,4, 2,5-2,9, .. $6,5-6,9)$ y un rango para $\mathrm{Mw} \geq 7,0$.

Para detallar la densidad regional de los sismos contenidos en el catálogo, se subdividió el área total de estudio en cuadrículas de $100 \mathrm{~km}^{2}$ y se contabilizaron los epicentros de los sismos por cada cuadrante (Mapa 2). Adicionalmente, se realizó un análisis geográfico, tomando en cuenta la energía sísmica (ES) liberada, la cual fue calculada a partir de la Mw de cada sismo. Para la determinación de la ES, se utilizó la ecuación propuesta por Gutenberg y Richter (1956). Así, se crearon cuadrantes individuales de $100 \mathrm{~km}^{2}$ en los cuales se sumó la ES liberada por cada uno de los eventos con $\mathrm{Mw} \geq 2,5$, ubicados en dicho cuadrante (Mapa 3, Figura 3).

Para el análisis de la sismicidad en cada cantón, se determinó la cantidad total de sismos por cantón, y, además, se calculó la densidad de sismos dividiendo la cantidad de sismos por el área de cada cantón. Si bien, la distribución de la sismicidad dentro de un mismo cantón no es uniforme, de manera general, estas relaciones permiten tener una visualización de qué tan sísmico resulta ser cada cantón y podría incluso servir para relacionarse con múltiples factores como la infraestructura y población en cada zona. Para el manejo general de los datos y la realización y presentación de los gráficos se utilizaron los programas Microsoft Office Excel, ArcMap 10.1, Adobe Illustrator CS6 y R.

\section{Análisis de resultados}

Siguiendo los conceptos planteados en la metodología de Step (1972), se realizó una inspección de la constancia de la cantidad de sismos a través del tiempo, según rangos de $\mathrm{Mw}$ a partir de 3,0 (Figura 1). Para 
cada gráfico se determinó la pendiente $(\mathrm{m})$, de forma que, si $\mathrm{m} \leq 0,1$ la tasa de sismicidad es considerada como constante durante el periodo analizado. Los resultados de esta inspección sugieren una $\mathrm{MC}$ de 5,0, ya que arriba de esta magnitud, $\mathrm{m}$ es casi cero, lo que implica muy poca variación de la tasa de sismicidad. Por el contrario, debajo de la MC de 5,0 la m es alta, mostrando poca constancia en la tasa de sismicidad y sugiriendo un catálogo incompleto. Por ejemplo, para el rango de $\mathrm{Mw}$ 4,0-4,9 la m es muy alta $(\mathrm{m}=6)$, es decir, se observa un aumento considerable en la detección de sismos a través del tiempo, especialmente después de 1990. Este aumento en la detección de sismos se debe a la mejora en la cobertura de estaciones a lo largo del tiempo, así como a las mejoras en las técnicas de detección y localización de sismos.

El resultado de una MC de 5,0 corresponde al periodo 1975-2014. Es probable que la MC sea mucho menor para periodos de tiempo posteriores a 1990, no obstante, su determinación ha quedado fuera del alcance de la presente investigación. Se recomienda su determinación mediante el método de Step (1972) en futuros estudios que analicen periodos de tiempo más específicos dentro del catálogo. 
Kevin Godinez-Rodríguez, Mario Arroyo-Solórzano, Lepolt Linkimer-Abarca. Geographic distribution of the earthquakes included in the catalog of National Seismological Network of Costa Rica

Figura 1: Cantidad de sismos según rangos de $\mathrm{Mw}$ en el periodo 19752014. A) $\mathrm{Mw}<3,0$. B) $\mathrm{Mw}$ de 3,0 a 3,9. C) Mw de 4,0 a 4,9. D) $\mathrm{Mw}$ de 5,0 a 5,9. E) Mw 6,0 a 6,9. F) Mw $\geq 7,0$. La línea discontinua denota el ajuste lineal de la cantidad de sismos en el tiempo y m representa la pendiente de dicho ajuste lineal en cada caso

A) $\mathrm{Mw}<3,0$

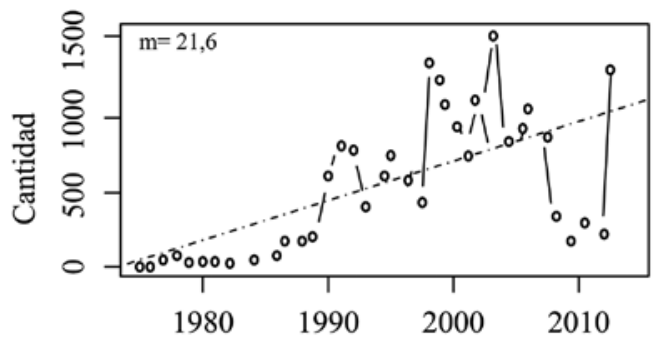

C) $4,0<\mathrm{Mw}<4,9$

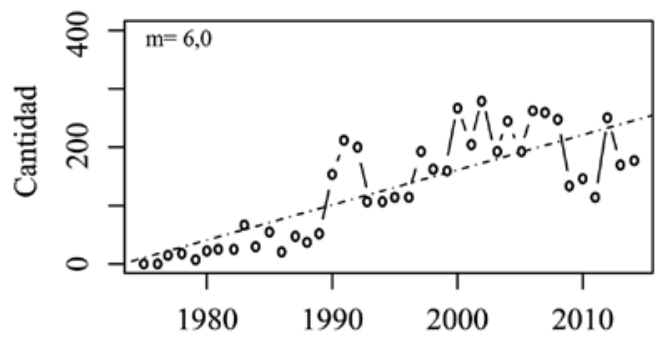

E) $6,0<\mathrm{Mw}<6,9$

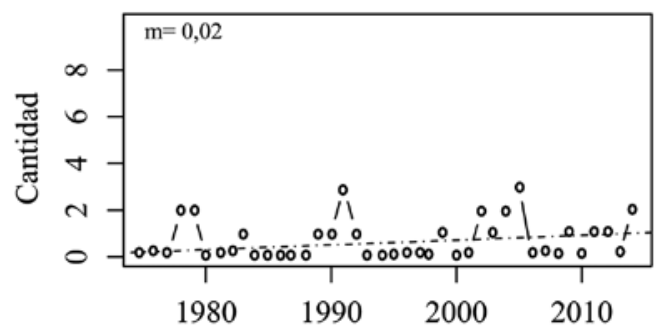

B) $3,0<\mathrm{Mw}<3,9$

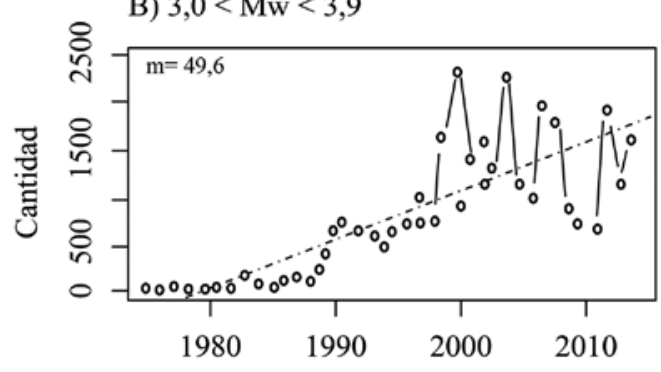

D) $5,0<\mathrm{Mw}<5,9$

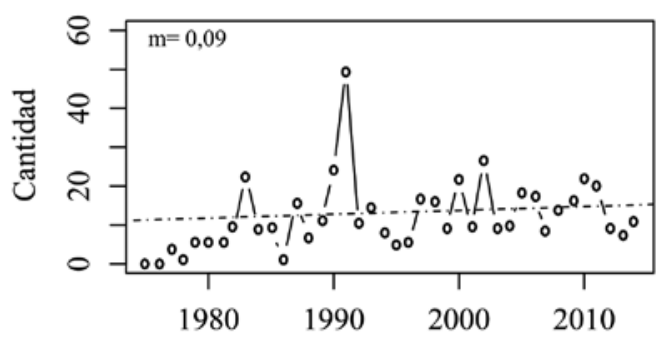

F) $\mathrm{Mw}>7,0$

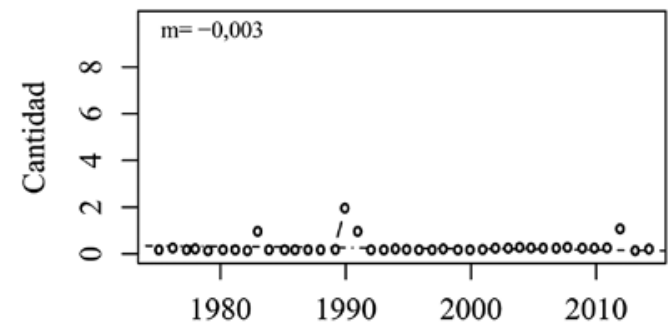

Fuente: Elaboración propia con base en el catálogo de sismos de la RSN. 


\section{Distribución geográfica de la sismicidad}

Se realizó un análisis espacio-temporal de la sismicidad de Costa Rica contenida en el catálogo de la RSN (Cuadro 1, Figura 2, Mapas 2, 3, 4 y 5). La Figura 2, muestra la distribución de la sismicidad subdividida en cuatro décadas para $\mathrm{Mw} \geq 5,0$ desde 1975 hasta el 2014. Esta figura permite visualizar las zonas con la sismicidad más alta en los periodos de tiempo señalados. En general, la sismicidad se concentra especialmente a lo largo de la costa Pacífica, asociada con la subducción de la placa del Coco bajo la placa Caribe y la microplaca de Panamá. En el periodo de observación, sobresalen los terremotos de Sámara de 1978 (Mw 6,8, Figura 2A), Golfito de 1983 (Mw 7,4, Figura 2A), Cóbano de 1990 (Mw 7,3, Figura 2B), Limón de 1991 (Mw 7,7, Figura 2B), Quepos de 1999 (Mw 6,9, Figura 2C) y Sámara del 2012 (Mw 7,6, Figura 2D).

La densidad regional de los sismos contenidos en el catálogo de la RSN se muestra en el Mapa 2. Los resultados obtenidos muestran que la sismicidad es abundante en la costa Pacífica de Costa Rica, especialmente entre la línea de costa y la Fosa Mesoamericana. En esta región se ubica, aproximadamente, la zona sismogénica interplacas de la subducción de la placa del Coco y han ocurrido terremotos importantes como: Sámara de 1978 (Mw 6,8), Golfito de 1983 (Mw 7,4), Cóbano de 1990 (Mw 7,3), Quepos de 1999 (Mw 6,9) y Sámara del 2012 (Mw 7,6, Figura 2). 
Kevin Godinez-Rodriguez, Mario Arroyo-Solórzano, Lepolt Linkimer-Abarca. Geographic distribution of the earthquakes included in the catalog of National Seismological Network of Costa Rica

Figura 2: Distribución geográfica de la sismicidad con $\mathrm{Mw} \geq 5,0$ por décadas durante 1975-2014. A) 1975-1984. B) 1985-1994. C) 19952004. D) 2005-2014

A) $1975-1984$

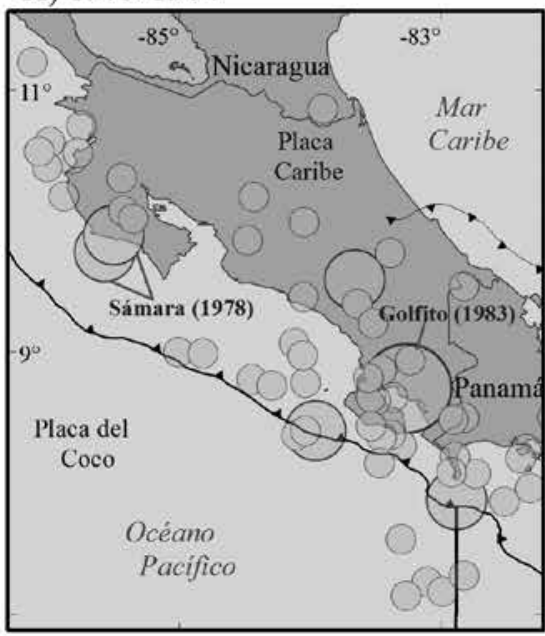

C) $1995-2005$

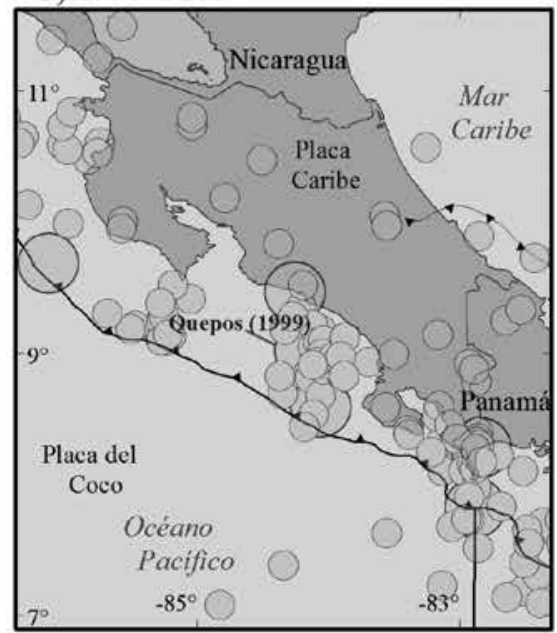

Magnitud (Mw)

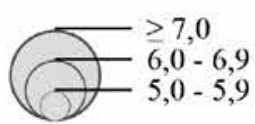

B) $1985-1994$

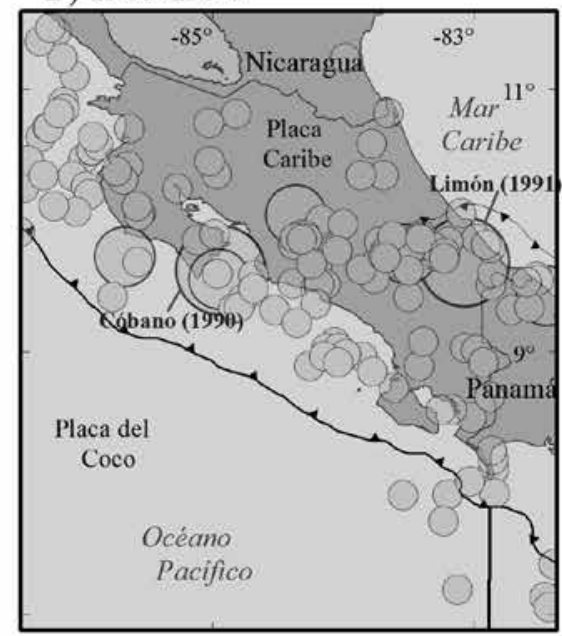

D) $2005-2014$
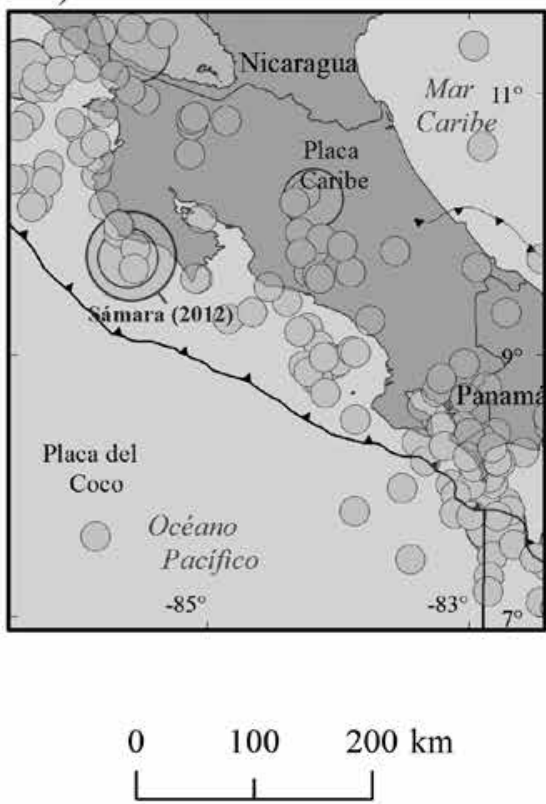

Fuente: Elaboración propia con base en el catálogo de sismos de la RSN. 
Otro límite de placas, que se resalta al mostrar la densidad de sismos, es el CDCCR. En el Mapa 2, se observa que la sismicidad es alta en casi la totalidad de las cuadrículas contenidas dentro del área propuesta por Montero y Rojas (2014) para el CDCCR. La alta densidad de sismos también expone la posición de las Fallas del Arco Volcánico (FAV) a lo largo de la cordillera de Guanacaste y del CDNP, en el sector Caribe, el cual fue el escenario del terremoto de Limón de 1991 (Mw 7,7, Figura 2B).

En particular, los resultados muestran una alta densidad de sismos en varios cúmulos (numerados con 1, 2 y 3), ubicados sobre la zona sismogénica interplacas del Pacífico Central, entre las penínsulas de Nicoya y Osa. Los cúmulos de alta sismicidad coinciden, aproximadamente, con la proyección hacia tierra de la subducción de montes submarinos de la placa del Coco, como lo son la cadena volcánica submarina de Fisher (cúmulos 1 y 2, Mapa 2) y el Plateau de Quepos (cúmulo 3, Mapa 2, Bilek, Schwartz y Deshon, 2003).

Otras dos regiones de alta sismicidad se ubican en la zona fronteriza entre Costa Rica y Panamá (cúmulos 4 y 5), aproximadamente, en la proyección hacia el norte de la Zona de Fractura de Panamá (Mapa 2). Estas observaciones sugieren que la subducción tanto de montes submarinos como de una falla transformada (ZFP) generan una alta deformación en la placa cabalgante, que resulta en la alta sismicidad concentrada en estas regiones específicas, tal como ha sido observada en estudios previos (e.g. Gardner y otros., 2001, Bilek y otros., 2003, Morell y otros., 2011).

Una alta concentración de sismos también sobresale en el mar Caribe (cúmulo 6, Mapa 2). Esta agrupación de sismos es denominada "nido de Parismina" y destaca por encontrarse en una zona de baja sismicidad que no está conectada directamente con un límite de placas. El origen de esta sismicidad puede estar relacionado con una falla local en el piso oceánico de la placa Caribe. 
Kevin Godinez-Rodríguez, Mario Arroyo-Solórzano, Lepolt Linkimer-Abarca. Geographic distribution of the earthquakes included in the catalog of National Seismological Network of Costa Rica

Mapa 2: Densidad de sismos con $\mathrm{Mw} \geq 2,5$ para áreas de $100 \mathrm{~km}^{2}$ en el periodo 1975-2014. El nombre de las estructuras tectónicas es señalado en el Mapa 1

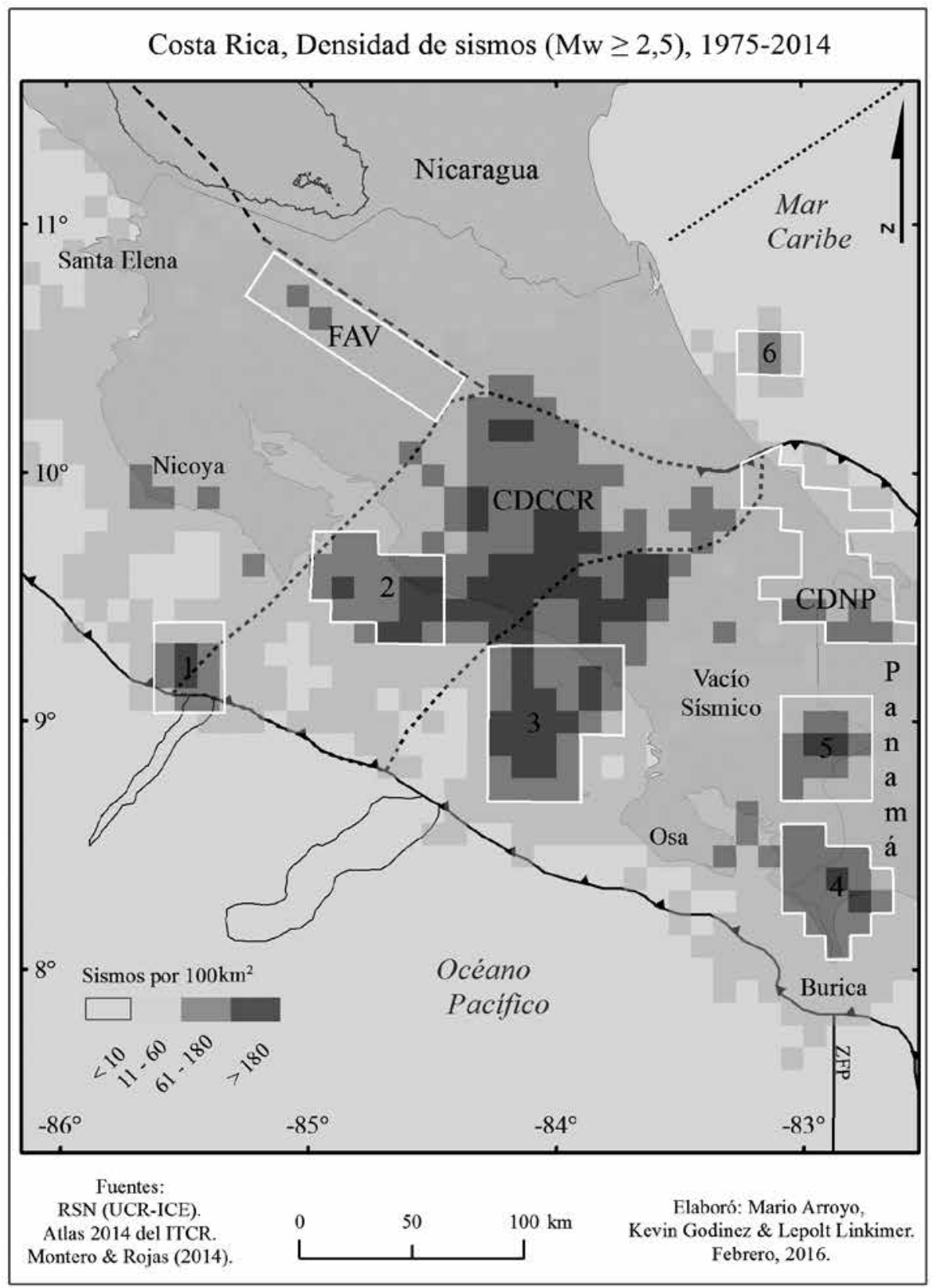


En Costa Rica existen también zonas con baja concentración de sismos durante el periodo de tiempo estudiado. La zona norte del país presenta una sismicidad muy baja, especialmente de sismos superficiales ( $<$ $30 \mathrm{~km}$ ). Además, en los alrededores de la península de Santa Elena, en donde a pesar de la ocurrencia de sismos de magnitud intermedia y alta, la zona posee una densidad de sismos baja en comparación con el resto del país. El área de baja sismicidad más llamativo, por su ubicación, es el localizado en el interior de Costa Rica al norte de la península de Osa. Esta área de quietud sísmica está rodeada por la zona sismogénica interplacas, el CDCCR, el CDNP y los cúmulos numerados como 3, 4 y 5 (Mapa 2). Geográficamente, este "vacío de sismos" corresponde con parte de la Cordillera de Talamanca, que puede ser descrito como una zona estable sismogénicamente durante el periodo de observación.

\section{Energía sísmica liberada}

La distribución geográfica de la energía sísmica (ES) liberada se muestra en el Mapa 3. Los seis cuadrantes con la mayor liberación de energía corresponden con la ubicación de los seis terremotos más grandes. Cinco de estos eventos se ubican en la zona sismogénica interplacas a lo largo de la costa pacífica, estos son los terremotos de Sámara de 1978 (Mw 6,8), Golfito de 1983 (Mw 7,4), Cóbano de 1990 (Mw 7,3), Quepos de 1999 (Mw 6,9) y Sámara del 2012 (Mw 7,6, Figura 2). El cuadrante con la mayor liberación de ES se ubica del lado Caribe, relacionado con la zona de deformación cortical del CDNP, en donde ocurrió el terremoto de Limón de 1991 (Mw 7,7, Figura 2). Además, a nivel cortical, también sobresalen cuatro terremotos en la zona del CDCCR, pero de menor Mw. Estos son los terremotos de Buenavista de 1983 (Mw 6,3), Alajuela de 1990 (Mw 5,9), Parrita del 2004 (Mw 6,2) y Cinchona del 2009 (Mw 6,1). Además, sobresale un terremoto al norte de la ZFP (Terremoto de Armuelles, 2003, Mw 6,5). 
Kevin Godinez-Rodríguez, Mario Arroyo-Solórzano, Lepolt Linkimer-Abarca. Geographic distribution of the earthquakes included in the catalog of National Seismological Network of Costa Rica

Mapa 3: Energía liberada por los sismos localizados por RSN a partir de Mw 2,5 en el periodo 1975-2014. Los sismos de Mw más alta se representan en forma de estrella. El nombre de las estructuras tectónicas es señalado en el Mapa 1

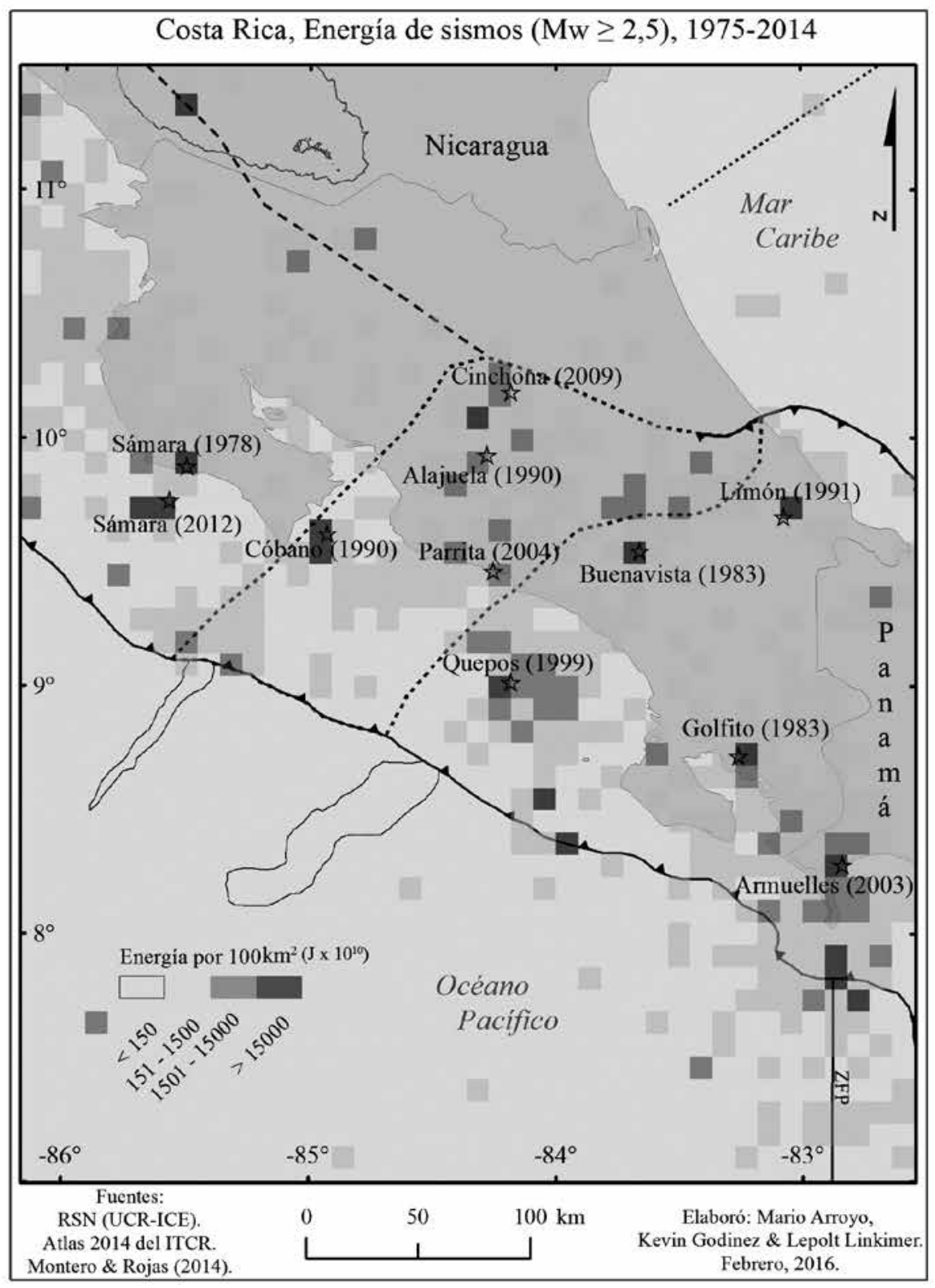


Kevin Godinez-Rodríguez, Mario Arroyo-Solórzano, Lepolt Linkimer-Abarca. Distribución geográfica de los sismos contenidos en el catálogo de la Red Sismológica Nacional de Costa Rica

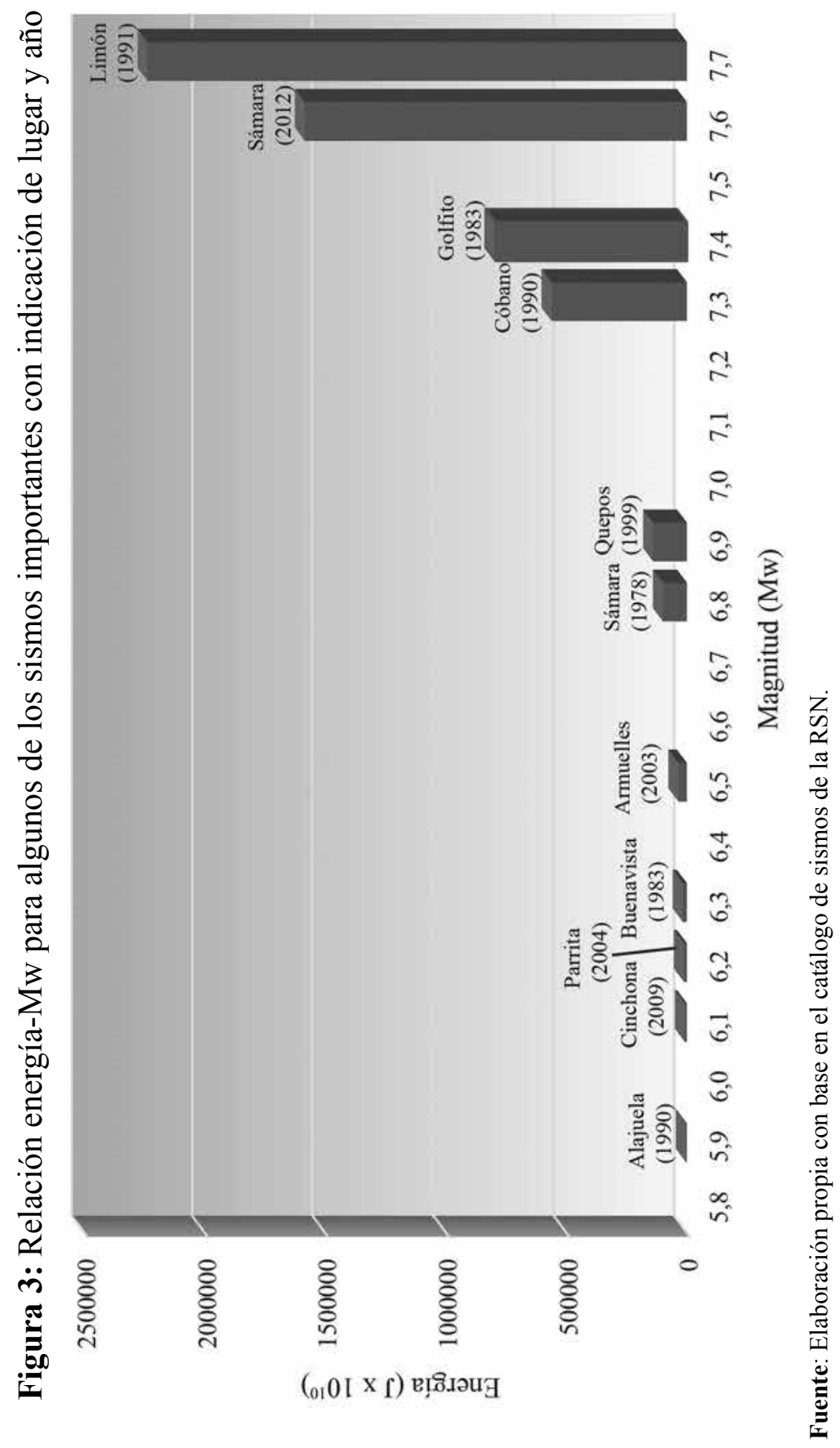


Kevin Godinez-Rodriguez, Mario Arroyo-Solórzano, Lepolt Linkimer-Abarca. Geographic distribution of the earthquakes included in the catalog of National Seismological Network of Costa Rica

Los resultados muestran que las zonas de mayor ES liberada durante el periodo 1975-2014 no corresponden exactamente con las zonas de mayor densidad de sismos mostradas en el Mapa 2. En particular, los cúmulos numerados con 1, 2, 4, 5 y 6 , no corresponden con zonas de alta liberación de energía, pese a que los sismos con $\mathrm{Mw}<6,0$ son muy frecuentes en estas áreas. Esto se debe a que la ES crece exponencialmente conforme aumenta la Mw (Figura 3). Con esto se muestra que las zonas donde más tiembla, no necesariamente son las zonas en donde se libera más ES.

Para visualizar esto con mayor facilidad, el sismo de Limón (1991) de Mw 7,7 liberó aproximadamente 501 veces más energía que el sismo de Alajuela (1990) de Mw 5,9, siendo la cantidad aproximada de ES liberada para cada uno de $2,24 \times 10^{16} \mathrm{~J}$ y $4,47 \times 10^{13} \mathrm{~J}$, respectivamente (Figura 3 ).

\section{Sismicidad por cantones}

Para analizar la sismicidad con base en la división político-administrativa de los cantones del país, se trabajó con todos los sismos de Mw $\geq 2,5$. Se omitieron todos los eventos que caen fuera del área terrestre de Costa Rica, es decir, los localizados en el mar, los cuales, tienen injerencia en los cantones costeros, sin embargo, la intención del análisis es enfocado en el espacio geográfico continental, el cual se encuentra dividido en cantones, a diferencia del espacio marítimo.

En el Mapa 4 y Figura 4, se aprecia la cantidad de sismos registrados en el periodo de estudio. Los cinco cantones con la sismicidad más alta son Pérez Zeledón, Parrita, Turrialba, Coto Brus y Quepos. Estos cantones se ubican principalmente en la zona central y sur del país y corresponden con zonas que además no han tenido una cobertura de estaciones óptima para el registro de sismos a lo largo de la historia de la RSN. Por otra parte, los cinco cantones con la sismicidad más baja son Flores, San Pablo, Tibás, Curridabat y Montes de Oca, todos estos ubicados en la parte central del país, la cual es justamente la que a lo largo de la historia ha contado con la mejor cobertura de estaciones sismológicas.

Los resultados anteriores de la cantidad de sismos por cantón, no reflejan necesariamente, las zonas con mayor sismicidad, ya que se puede notar que los cantones con más sismos están caracterizados por áreas grandes en comparación con los cantones con menos sismos. Por esta razón, se presentan, además, los resultados usando la densidad de sismos por cantón en el Mapa 5 y Figura 4. 
Kevin Godinez-Rodríguez, Mario Arroyo-Solórzano, Lepolt Linkimer-Abarca. Distribución geográfica de los sismos contenidos en el catálogo de la Red Sismológica Nacional de Costa Rica

Mapa 4: Cantidad total de sismos con $\mathrm{Mw} \geq 2,5$ por cantón y lista de los 10 cantones con la mayor y menor cantidad de sismos localizados por la RSN. El nombre de las estructuras tectónicas es señalado en el Mapa 1

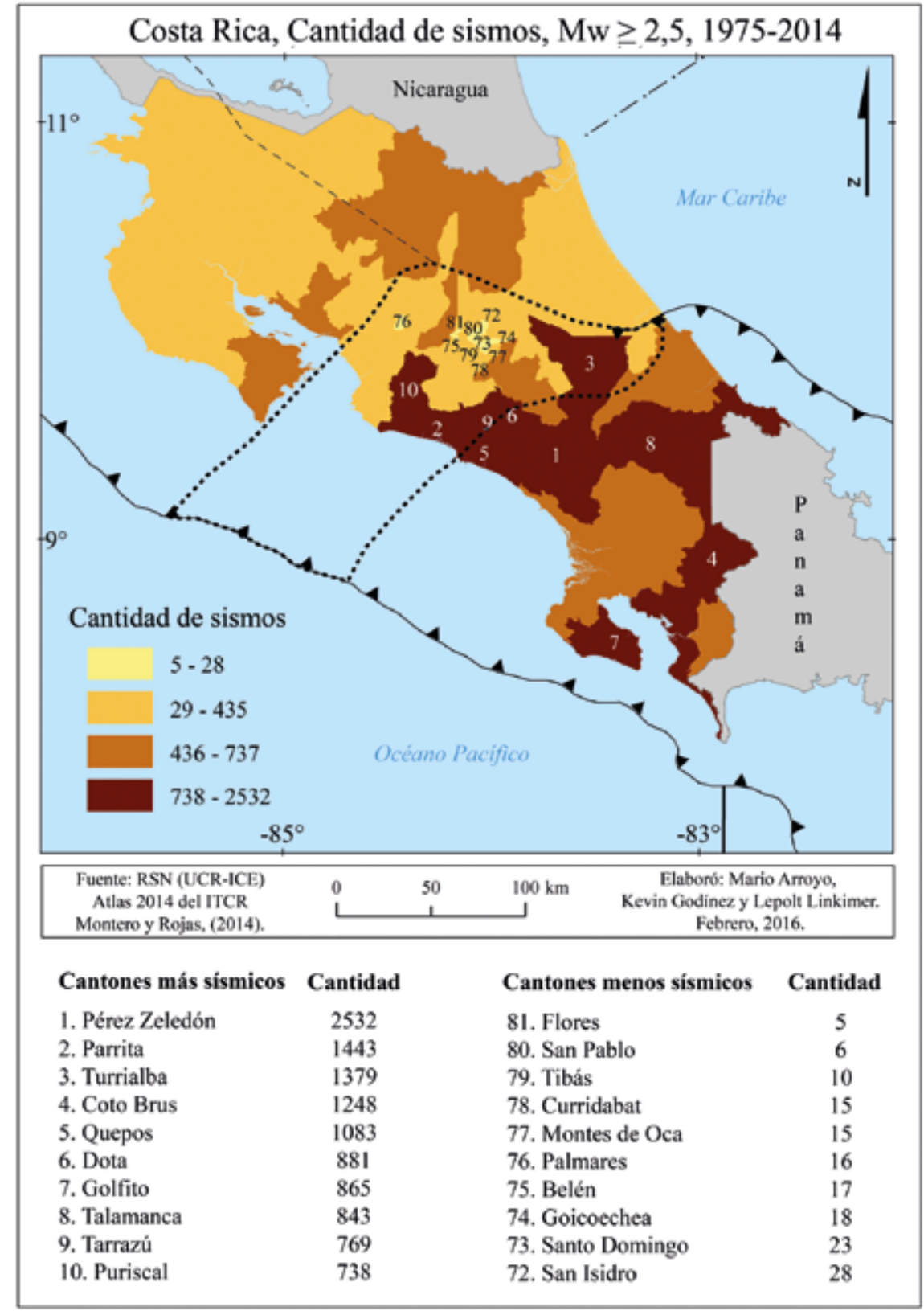


Kevin Godinez-Rodríguez, Mario Arroyo-Solórzano, Lepolt Linkimer-Abarca. Geographic distribution of the earthquakes included in the catalog of National Seismological Network of Costa Rica

Los resultados muestran como los cantones con la densidad más alta de sismos son Parrita, El Guarco, Tarrazú, León Cortés y Desamparados, todos ubicados en la zona Central y del Pacífico Central del país, y con una densidad mayor a 2,4 sismos $/ \mathrm{km}^{2}$. La sismicidad alta de esta zona, es el resultado de la cercanía a la zona sismogénica interplacas, y, además, a la presencia de las fallas del CDCCR. Por otra parte, los cantones con la menor densidad de sismos son Los Chiles, Guácimo, Pococí, La Cruz y Liberia. En estos casos todos están ubicados en la zona Norte o Pacífico Norte, con una densidad menor a 0,14 sismos $/ \mathrm{km}^{2}$.

La mayoría de los sismos que se localizan en Costa Rica no son percibidos por la población, debido a su magnitud baja o su localización en zonas poco pobladas. Por esta razón, la cantidad y densidad de sismos por cantón no necesariamente corresponde con una mayor percepción de la sismicidad en dichas zonas. En relación con los sismos que han sido reportados como sentidos a la RSN (Linkimer y Alvarado, 2014), los cantones de Pérez Zeledón, Turrialba y El Guarco han sido el escenario de la mayor cantidad de estos sismos sentidos. Esto concuerda con los resultados obtenidos que muestran que, Pérez Zeledón y Turrialba son cantones con alta cantidad de sismos y El Guarco un cantón con una densidad de sismos alta (Figura 4). 
Kevin Godinez-Rodríguez, Mario Arroyo-Solórzano, Lepolt Linkimer-Abarca. Distribución geográfica de los sismos contenidos en el catálogo de la Red Sismológica Nacional de Costa Rica

Mapa 5: Densidad de sismos con $\mathrm{Mw} \geq 2,5$ por cantón localizados por la RSN en el período 1975-2014. El nombre de las estructuras tectónicas es señalado en el Mapa 1

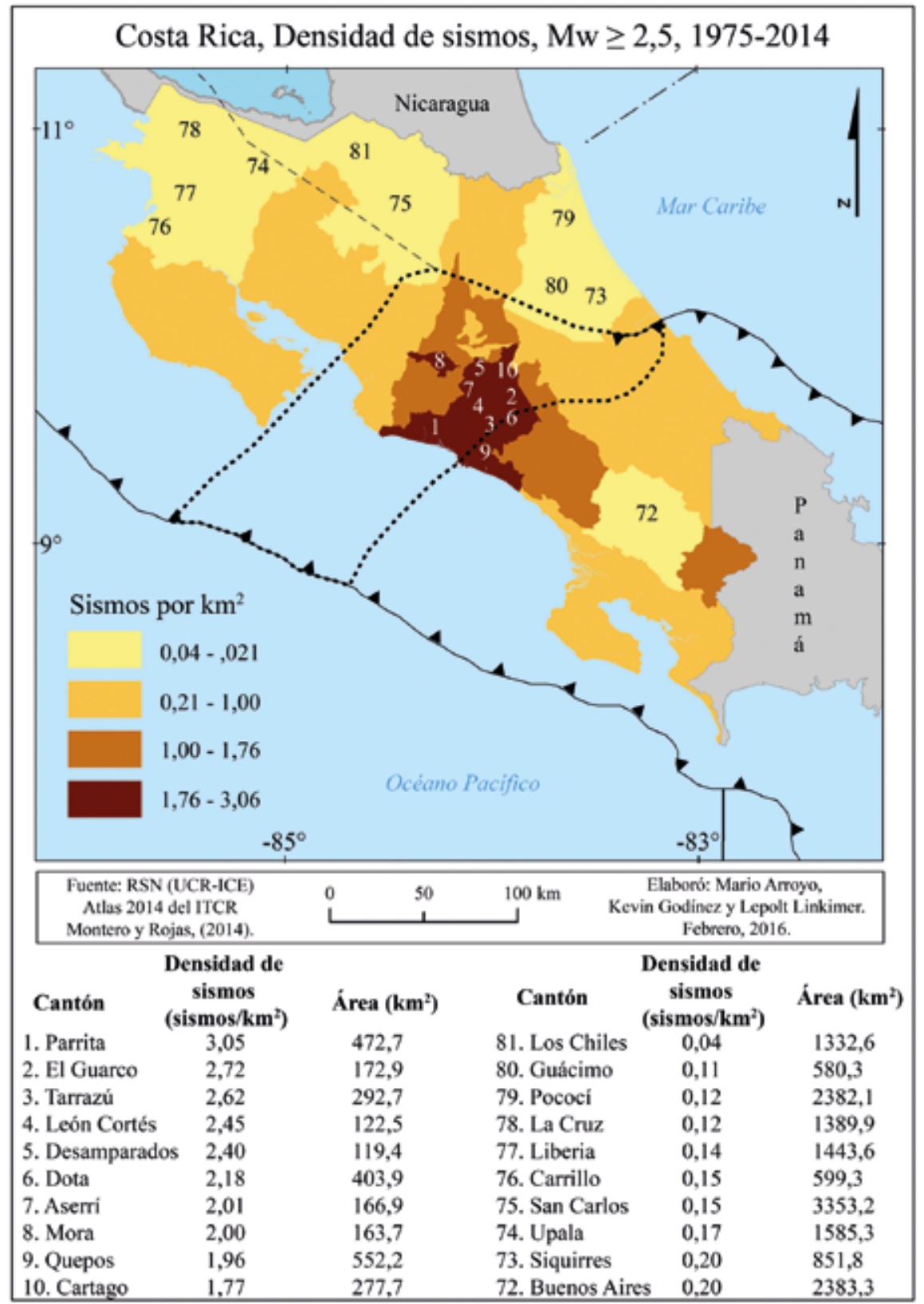


Kevin Godinez-Rodríguez, Mario Arroyo-Solórzano, Lepolt Linkimer-Abarca. Geographic distribution of the earthquakes included in the catalog of National Seismological Network of Costa Rica

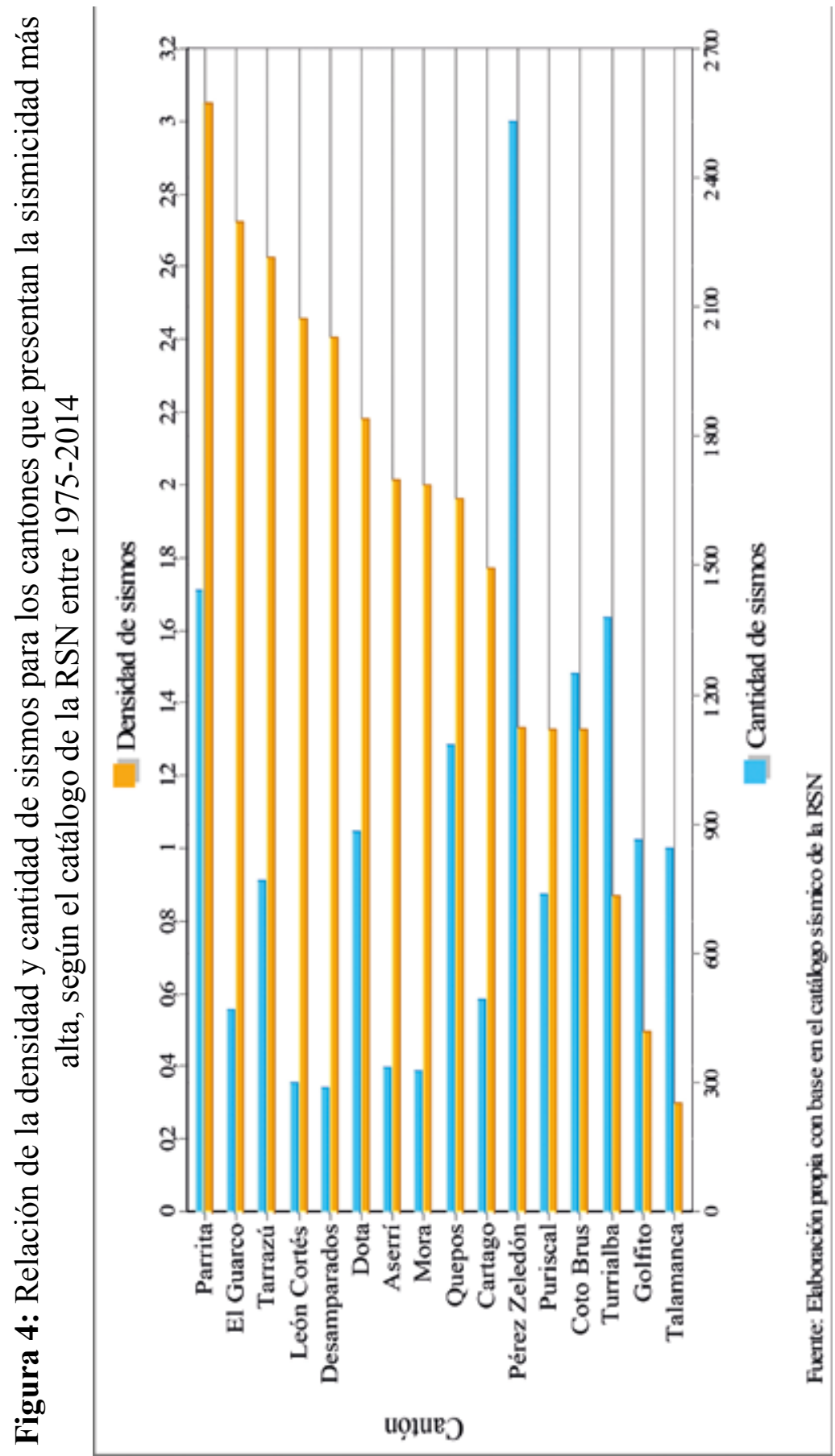




\section{Conclusiones}

En este estudio se analizó el catálogo sísmico de la RSN compuesto por 111.850 sismos ocurridos entre el 4 de noviembre de 1974 y el 31 de diciembre del 2014. La inspección de la constancia en el tiempo de diversos rangos de Mw sugiere que este catálogo tiene una MC de 5,0 para el periodo 1975-2014. Este valor debería ser corroborado con el uso de otras metodologías y para rangos de tiempo más específicos.

El análisis espacio-temporal de la sismicidad de Costa Rica reveló que la sismicidad es abundante en la costa Pacífica del país, especialmente, entre la línea de costa y la Fosa Mesoamericana, asociada con la subducción de la placa del Coco bajo la placa Caribe y la microplaca de Panamá. Otras estructuras que se resaltan al graficar la densidad de sismos son el CDCCR, las FAV a lo largo de la cordillera de Guanacaste y del CDNP, en el sector Caribe.

En particular, los resultados muestran una alta densidad de sismos en varios cúmulos ubicados sobre la zona sismogénica interplacas del Pacífico Central, entre las penínsulas de Nicoya y Osa, ubicados aproximadamente en la proyección hacia tierra de la cadena volcánica submarina de Fisher y el Plateau de Quepos, y, además, se presentan cúmulos de alta sismicidad en la zona fronteriza entre Costa Rica y Panamá, aproximadamente, en la proyección hacia el norte de la ZFP. Estas observaciones sugieren que la subducción tanto de montes submarinos como de una falla transformada (ZFP) genera una alta deformación en la placa cabalgante, que resulta en la alta sismicidad concentrada en estas regiones específicas. Una alta concentración de sismos también resalta el llamado "nido de Parismina", que puede estar relacionado con una falla local en el piso oceánico de la placa Caribe.

La zona de baja sismicidad más llamativa coincide geográficamente con parte de la cordillera de Talamanca, y está rodeada de zonas de alta sismicidad como la zona sismogénica interplacas, el CDCCR y el CDNP. Esta parte de la cordillera de Talamanca puede ser descrita como una zona estable sismogénicamente durante el periodo de observación analizado.

Los resultados muestran que las zonas de mayor ES liberada durante el periodo 1975-2014 no corresponden exactamente con las zonas de mayor densidad de sismos. Esto se debe a que la ES crece exponencialmente conforme aumenta la Mw. Las áreas con la mayor liberación de ES se 
Kevin Godinez-Rodríguez, Mario Arroyo-Solórzano, Lepolt Linkimer-Abarca. Geographic distribution of the earthquakes included in the catalog of National Seismological Network of Costa Rica

ubican en la zona sismogénica interplacas, a lo largo de la costa pacífica, en donde ocurrieron los terremotos de Sámara de 1978 (Mw 6,8), Golfito de 1983 (Mw 7,4), Cóbano de 1990 (Mw 7,3), Quepos de 1999 (Mw 6,9) y Sámara del 2012 (Mw 7,6). El sector con la mayor liberación de ES se ubica del lado Caribe, relacionado con el CDNP, en donde ocurrió el terremoto de Limón de 1991 (Mw 7,7). A nivel cortical, sobresalen dentro del CDCCR cuatro zonas con una alta liberación de ES, en los sitios donde ocurrieron los terremotos de Buenavista de 1983 (Mw 6,3), Alajuela de 1990 (Mw 5,9), Parrita del 2004 (Mw 6,2) y Cinchona del 2009 (Mw 6,1).

La inspección de la sismicidad, considerando la división políticaadministrativa del país, muestra que los cantones con la cantidad más alta de sismos son Pérez Zeledón, Parrita, Turrialba, Coto Brus y Quepos, y con la densidad más alta de sismos son Parrita, El Guarco, Tarrazú, León Cortés y Desamparados. Por otro lado, los cantones con la cantidad más baja de sismos son Flores, San Pablo, Tibás, Curridabat y Montes de Oca, y con la menor densidad de sismos son Los Chiles, Guácimo, Pococí, La Cruz y Liberia.

\section{Agradecimientos}

Agradecemos a todo el personal profesional, técnico y administrativo tanto en la UCR como en el ICE, que han colaborado con la RSN a lo largo de más de 30 años, y que han permitido el registro y localización de los sismos contenidos en el catálogo analizado. Este trabajo ha sido posible gracias a los recursos económicos que la RSN recibe provenientes de la Ley Nacional de Emergencias $\mathrm{N}^{\mathrm{o}} 8488$ y los recursos económicos y de personal brindados por la UCR, en particular el apoyo de la Rectoría a través de horas asistente. Este estudio resulta, en primera instancia, de una iniciativa de investigación como parte de una Práctica Profesional Supervisada de la carrera en Ciencias Geográficas de la Universidad Nacional (UNA), por lo cual, agradecemos al personal docente de la Escuela de Ciencias Geográficas de la UNA que nos apoyó en la realización del mismo. Este artículo es también el resultado de los proyectos de investigación Vigilancia sísmica de Costa Rica (113-B5-704) y Estudio del catálogo de sismos de la Red Sismológica Nacional de Costa Rica (113-B5-A02) inscritos ante la Vicerrectoría de Investigación de la Universidad de Costa Rica. 


\section{Referencias}

Benito, M. (2008). Evaluación regional de la amenaza sísmica en Centro América. Informe final de Proyecto con América Latina 2008. Univ. Politécnica de Madrid, España. (pp. 84).

Benito, M. y Jiménez, E. (1999). Peligrosidad sísmica. En: Física de la Tierra, 11, 13-47.

Bilek, S. L., Schwartz, S. y Deshon, H. (2003). Control of seafloor roughness in earthquake rupture behavior. En: Geology, 31, 455-458.

Bolt, B. (1993). "Earthquake". University of California, Berkeley. New York: W.H. Freeman and Company.

Caneva, A., Salcedo, E., Van Heissenoven, R. y Alfaro, A. (2003). Estudio de sismicidad regional para el análisis de la amenaza sísmica para Bogotá. En: Ingeniería y Universidad. 7, N (2),133-150.

Cárdenas, C., Garzón, Y., Santa, L. y Castillo, L. (2010). Modelo de Poisson para la ocurrencia y magnitud espacio-temporal de los sismos en Colombia. En: UD y la Geomática. (4), 1-52.

Chouliaras, G. (2009). Investigating the earthquake catalog of the National Observatory of Athens. En: National Hazards Earth System Science.(9), 905-912.

Fan, G., Beck, S. y Wallace, T. (1993). The seismic source parameters of the 1991 Costa Rica aftershock sequence: Evidence for a transcurrent plateboundary. En: J. Geophys. Res., 98, 15759-15778.

Gardner, T., Marshall, J., Merritts, D., Bee, B., Burgette, R., Burton, E., Cooke, J., Kehrwald, Protti, M., Fisher, D. y Peter, S. (2001). Holocene forearc block rotation in response to seamount subduction, southeastern Peninsula de Nicoya, Costa Rica. En: Geology, 29, 151-154.

Goes, S., Velazco, A. y Schwartz, S. (1993). The April 22, 1991, Valle de la Estrella, Costa Rica (MW 7.7) earthquake and its tectonic implications. A broadband study. En: J. Geophys. Res. 98,8127-8142.

Gutenberg, B. y Richter, C. (1956): Earthquake Magnitude, Intensity, Energy and Acceleration (second paper). En: Bulletin of the Seismological Society of America. 46,(2), 105-145.

Linkimer, L. y Alvarado, G. (2014). Distribución espacio-temporal de la sismicidad sentida en Costa Rica (1976-2013) en el marco histórico del 30 aniversario (1982-2012) de la Red Sismológica Nacional 
Kevin Godinez-Rodriguez, Mario Arroyo-Solórzano, Lepolt Linkimer-Abarca. Geographic distribution of the earthquakes included in the catalog of National Seismological Network of Costa Rica

(RSN: UCR-ICE). En: Revista Geológica de América Central. N ${ }^{\circ}$ Especial: 30 Aniversario, 45-71\}.

Lücke, O. (2012). Moho structure of Central America based on three-dimensional lithospheric density modelling of satellite-derived gravity data. En: . (103), 1733-1745.

Marshall, J. S., Fisher, D. M. y Gardner, T. (2000): Central Costa Rica deformed belt: kinematics of diffuse faulting across the western Panama block. En: Tectonics, 19 (3), 468-492.

Mignan, A., y Woessner, J. (2012). Estimating the magnitude of completeness for earthquake catalogs. En: Community Online Resource for Statistical Seismicity Analysis. Recuperado de: http://www.corssa. org., DOI:10.5078/corssa-00180805.

Montero, W. (2001): Neotectónica de la región central de Costa Rica: frontera oeste de la microplaca de Panamá. En: Revista Geológica de América Central. 24, 29-56.

Montero, W. y Rojas, W. (2014). Las fallas Picagres y Purires y su relación con la secuencia sísmica de Puriscal de 1990. En: Revista Geológica de América Central, (50), 39-69.

Morales, L. (1986): Historia de la Sismología en Costa Rica. En: Revista de Filosofía de la Universidad de Costa Rica, 24, ${ }^{\circ}$ (59), 93-104.

Morell, K. D., Fisher, D. M., Gardner, T. W., La Femina, P., Davidson, D. y Teletzke, A. (2011). Quaternary outer fore-arc deformation and uplift inboard of the Panama Triple Junction, Burica Peninsula. En: J. Geophys. Res., 116, B05402, doi: 10.1029/2010JB007979.

Ranjit, D., Wason, H. y Sharma, M. (2012). Temporal and spatial variations in the magnitude of completeness for homogenized moment magnitude catalogue for Northeast India. En: Journal of Earth System Science, $\mathrm{N}^{\circ}$ (121), 19-28.

Rojas, W. (1993). Catálogo de Sismicidad Histórica y Reciente en América Central: Desarrollo y Análisis. (Tesis de Licenciatura. Escuela Centroamericana de Geología. Universidad de Costa Rica).

Rojas, W., Cowan, H., Lindholm, C., Dahle, A. y Bungum, H. (1993). Regional Seismic Zonation for Central America A Preliminary Model. Informe Norsar, Norway, Dec. 1993.

Segura, J., Quintero, R., Burgoa, B., Jiménez, W. (2014). Análisis de la actividad sísmica en Costa Rica durante el 2010 y resumen de los 
eventos sísmicos más importantes presentados en Costa Rica de 1983 - 2012. Revista Geográfica de América Central. (52). 71-102.

Spence, W., Sipkins, S. y Choy, G. (1989). Measuring the size of an earthquake. En: Earthquakes and Volcanoes. 21, (1), 58-63.

Stepp, J. (1972). Analysis of Completeness of the Earthquake Sample in the Puget Sound Area and its Effect on Statistical Estimates of Earthquake Hazard. En: Caneva, A., Salcedo, E., Van Heissenoven, R. y Alfaro, A. (2003). Estudio de sismicidad regional para el análisis de la amenaza sísmica para Bogotá. En: Ingeniería y Universidad. 7, (2), 133-150.

Valladares, R. (2013). Determinación de parámetros sísmicos asociados con la amenaza sísmica en la región nororiental de Venezuela. (Tesis de Licenciatura. Escuela de Ciencias, Departamento de Física. Universidad de Oriente Núcleo de Sucre, Cumana, Venezuela)

Woessner, J. y Wiemer, S. (2005). Assessing the quality of earthquake catalogues: estimating the magnitude of completeness and tts uncertainty. En: Bulletin of the Seismological Society of America. 95,(2), 684-698. 